\title{
Kinematic analysis of the head by inertial sensors. Test-retest reproducibility and clinical use feasibility ${ }^{\star}$
}

\author{
Raphaël Portero ${ }^{1}$, Jean-Pierre Bleton ${ }^{2,3}$, Dominique Garric $^{1}$, Ingrid Masson ${ }^{1}$, Serge Mesure ${ }^{3}$ and Pierre Portero ${ }^{1,4}$ \\ 1 BIOTN - EA 7377, Université Paris-Est Créteil, Faculté de Médecine, Créteil, France \\ 2 Unité James Parkinson, Service de Neurologie, Fondation OPH Rothschild, Paris, France \\ 3 UMR 7287 CNRS, Institut des Sciences du Mouvement, Aix-Marseille Université, Marseille, France \\ 4 Service de Rééducation Neuro-Orthopédique, Hôpital Rothschild (AP-HP), Paris, France
}

Received 19 March 2015 - Accepted 29 September 2015

\begin{abstract}
The aim of this study was to evaluate the reproducibility of a protocol using inertial sensors in order to characterize primary and associated movements of the head. Twenty-two subjects were evaluated twice in the same experimental conditions (3 days interval). Two inertial sensors allowed the evaluation of the range of movement of the head. Three complete cycles of movements were realised in each plane. A patient suffering from cervical dystonia was also evaluated with the same protocol before and after self-training program. Results show a good reproducibility for all ranges of movement except for the associated movements realised in the sagittal plane. Thereafter, our protocol allowed us to notice an improvement of the kinematics of the head after the self-training program followed by the patient. These results show the reproducibility of the inertial sensors as evaluation tools for head movements, a change in the proposed method will allow a generalization to cervical movements.
\end{abstract}

Key words: Head kinematics, inertial sensors, primary movements, associated movements, cervical dystonia, rehabilitation

Résumé. Évaluation de la cinématique de la tête grâce à l'utilisation de capteurs inertiels. Reproductibilité et faisabilité en utilisation clinique.

Le but de cette étude était d'évaluer la reproductibilité d'un protocole utilisant des capteurs inertiels afin de caractériser les mouvements primaires et associés de la tête. Vingt-deux sujets sains ont été évalués deux fois à trois jours d'intervalle dans les mêmes conditions expérimentales. Deux capteurs inertiels ont permis l'évaluation de l'amplitude articulaire de la tête. Trois cycles de mouvements complets dans chaque plan ont été réalisés. Un sujet atteint de dystonie cervicale a également été évalué à l'aide du même protocole avant et après exercices d'auto-rééducation. Les résultats montrent que toutes les amplitudes sont reproductibles sauf pour les mouvements associés dans le plan sagittal. Ensuite, notre protocole a permis d'observer une amélioration de la cinématique de la tête après les exercices d'auto-rééducation effectués par le patient. Ces résultats montrent la fiabilité des capteurs inertiels comme outils d'évaluation des mouvements de la tête, une évolution de la méthode proposée permettra une généralisation aux mouvements cervicaux.

Mots clés : Cinématique de la tête, capteurs inertiels, mouvements principaux, mouvements associés, dystonie cervicale, rééducation

\section{Introduction}

Structural and functional characteristics of the head-neck segment make this area exposed to overwork or move-

\footnotetext{
* Le matériel de cet article a fait l'objet d'une présentation au $14^{\mathrm{e}}$ congrès de la SOFAMEA qui a eu lieu à Genève du 4 au 6 février 2015.
}

ment disorders responsible for neck pain and posturaldisorders. Indeed, cervical function, and particularly range of motion of head movements (RoM), could be altered by localised disorders, aging, and some activities (Bertuit, Van Geyt, \& Feipel, 2008; Portero 2009). Thus, analysis of 3-D head kinematics in the three planes (sagittal, frontal, and transverse), particularly global head 
RoM, is relevant to characterize pathologies and for evaluating the effects of rehabilitation programs (Prushansky \& Dvir, 2008).

Various measurement devices have been used to characterize head movements such as the cervical range of motion (CROM) device or universal inclinometer (Audette, Dumas, Côté, \& De Serres, 2010), ultrasound-based sensors (Zebris) (Dvir \& Prushansky, 2000), electromagnetic tracking (Morphett, Crawford, \& Lee, 2003), goniometer and electrogoniometer (Feipel, Rondelet, Le Pallec, \& Rooze, 1999; Salvia, Champagne, Feipel, Rooze, \& de Beyl, 2006; Youdas et al., 1992), and optical-based systems (Castro, Sautmann, Schilgen, \& Sautmann, 2000). Recently, studies have focused on the use of inertial sensors for characterizing kinematics of patients' cervical spine. Theobald, Jones, and Williams (2012) concluded, in a rehabilitation study, that these sensors represent a reliable method for quantifying cervical RoM in the 3D space, and Duc, Salvia, Lubansu, Feipel, and Aminian (2013) demonstrated their usefulness for evaluating the cervical spine mobility after surgery. Indeed, their small size, high sample rate, and easy use make inertial sensors a useful tool for evaluating patients or healthy subjects.

Thus, the protocol proposed by Theobald et al. (2012) evaluates the reliability thanks to a repeatability study. Considering the effects of learning process when two tests are performed in a too short period (Hopkins, 2000), a classical test-retest study should indeed be performed in order to assess the reliability of the inertial sensors. However in Theobald's study (2012), the order of movements in the 3-D space was not randomized although the mechanical properties of musculo-skeletal tissues of the head-neck segment depend on the order of performed movements. As a matter of fact, a cervical RoM changes according to its position in the protocol, so that randomization of movements seems fundamental (McNair, Portero, Chiquet, Mawston, \& Lavaste, 2007).

The head movements could be broken up into a primary and two associated movements due to the cervical spine kinematics (Trott, Pearcy, Ruston, Fulton, \& Brien, 1996) and their quantifications give an inside view into the kinematics of the head-neck segment for healthy subjects (Feipel et al., 1999; Trott et al., 1996). For example, the primary movement of lateral flexion is combined with associated movements in the two other planes. In order to quantify the RoM of the primary and of the two associated movements, the cardan representation, that divides a 3 -D rotation into three successive rotations around anatomical axis, is used to describe 3-D joint movements in an intuitively interpretable way (Begon \& Lacouture, 2005). However, given the fact that composition of rotations is not commutative in the 3-D space, the angular values can be different depending on the chosen rotation order, so called cardan sequence (Karduna, McClure, \& Michener, 2000). The International Society of Biomechanics (ISB) has recommended a sequence for motion between adjacent vertebrae (Wu, 2002), but not for the whole cervical spine (Boussion, 2008). Moreover, some au- thors chose various sequences in function of the primary movement performed (Bonnechère et al., 2014; Watier 2006). Nevertheless, in our knowledge, there is no reproducibility of values of RoM for the primary and associated movements estimated through the cardan representation.

Evaluation of primary and associated RoM could be also useful for clinical applications (Feipel et al., 1999), and particularly in complex movement disorders such as cervical dystonia (CD), such as defined by Albanese et al. (2013). CD is a syndrome characterized primarily by unwanted muscle spasms giving rise to involuntary movements and abnormal postures of the head. In CD, like in all localizations of dystonia, it appears that inhibition is defective, leading to a decrease of muscular selectivity and a muscular overflow. Loss of reciprocal inhibition can be partly responsible for the co-contraction of agonist and antagonist muscles that characterizes voluntary movements in dystonia (Hallett, 2011). CD increases the complexity of biomechanical characteristics of the head movements. Currently, the best treatment option for CD is injection of botulinum neurotoxin (BoNT) into the affected muscles (Marsh, Monroe, Brin, \& Gallagher, 2014). Rehabilitative approaches are generally associated as a complementary treatment despite the lack of any scientific evidence to support claims of their effectiveness and it could be relevant to investigate their effects on primary and associated RoM.

The main goal of our study was to examine the testretest reproducibility for evaluating head range of primary and associated movements with a cardan method using inertial sensors. We hypothesized that there are no significant differences of head range of primary and associated movements in the 3-D space between test and retest results for the healthy population. The secondary goal was to use these inertial sensors in a case study of CD. We hypothesized that the inertial sensors are efficient tools for characterizing the clinical features and for appreciating the efficiency of a tailored retraining program for CD patient.

\section{Method}

\subsection{Population}

For the test-retest reproducibility study, twenty-two healthy subjects $(27.8 \pm 7.0$ years, $73.1 \pm 16.7 \mathrm{~kg}$, and $173.8 \pm 8.8 \mathrm{~cm}$ ) volunteered to participate in the study. The procedures of the study complied with the Declaration of Helsinki and subjects have been provided informed consent.

For the clinical application, we have studied the case of a CD patient chosen randomly among the outpatients of the dystonia consultation of the Foundation OPH Rothschild (Paris). The male patient aged 44 had a one year history of CD: left torticollis, left laterocollis, retrocollis and right lateral shift, Toronto Western Spasmodic Torticollis Scale 58/85 (Consky, Basinski, Belle, 
Ranawaya, \& Lang, 1990). The patient received his last injection of BoNT three months before.

\subsection{Equipment}

Two wireless inertial sensors (model $i 4$ Motion ${ }^{\circledR}$, TechnoConcept, France) were placed on the forehead and on the trunk (at the level of the xiphoid process) of the subjects in order to measure the head and the trunk kinematic parameters. Locations of the sensors were adapted from the study of Boussion, Bahuaud, and Chèze (2011). The sensors were relatively small $(49 \mathrm{~mm}(\mathrm{~h}) \times 38 \mathrm{~mm}$ $(\mathrm{w}) \times 19 \mathrm{~mm}(\mathrm{~d}))$ and light $(0.025 \mathrm{~kg})$, whilst containing three integrated sensing elements in each orthogonal axis: a gyroscope (maximum angular velocity: $500^{\circ} \cdot \mathrm{s}^{-1}$ ), an accelerometer (maximal linear accelerations: $6 \mathrm{~g}$ ), and a magnetometer (maximal local magnetic field: $\pm 6 \mathrm{G}$ ). The TechnoConcept ${ }^{\circledR}$ datasheet reports accuracy inferior to $1^{\circ}$ during static testing and of $\pm 1^{\circ}$ during dynamic testing. Kinematic data were collected at $100 \mathrm{~Hz}$.

\subsection{Experimental procedure}

In a first step, for validating the method with the inertial sensors, we have applied the following protocol, named RoMP (RoM Protocol). Tests were performed in a quiet room. Subjects were seated on a rigid seat with hips and knees at $90^{\circ}$ and hands behind the back as suggested by Demaille-Wlodyka et al. (2007) (Fig. 1). The feet were flat on the ground and parallel. The heels were placed against the legs of the chair. The head reference position was self-determined by the natural, comfortable anatomical position of the subject when his/her gaze was horizontal. The vertical position of his/her eyes in the seated position was measured and marked on a wall with a target placed at three meters.

After being placed in the reference position (i.e. seated and gaze on the target), the subjects closed their eyes as suggested in previous studies (Dvir \& Prushanski, 2000; Demaille-Wlodyka et al., 2007) and had to perform three complete and continuous cycles of the head in the maximal RoM respectively in the three anatomical planes (flexion-extension, right and left lateral flexion, right and left axial rotation). These movements had to be performed with neither trunk or shoulder compensatory movements, nor pain or discomfort. The movement order was randomized by drawing lots. Subjects realized the RoMP twice (intervals between test (T1) and retest (T2) was $3.1 \pm 1.5$ days) in the same experimental conditions.

In a second step, we have applied the experimental RoMP to a single case. Patient voluntary head movements were recorded before and after a 20-minute self-training of tailored exercises (Bleton, 2010). The self-training program consisted of constant repetition of the antagonist muscles activation, which acts to turn the head to the opposite side than the CD one. Records were performed

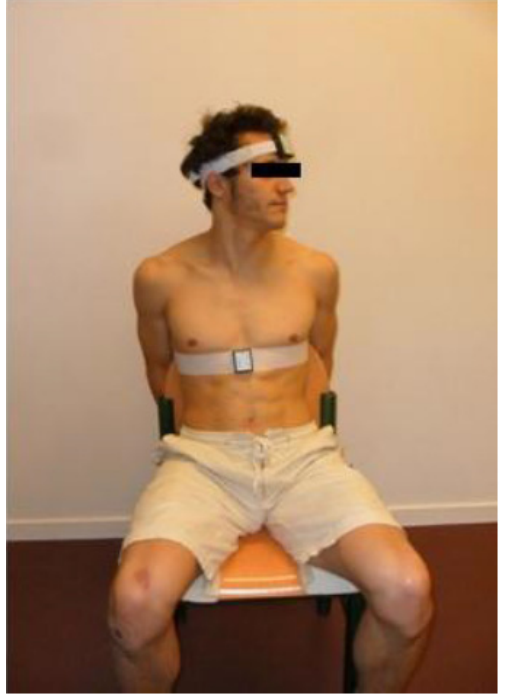

Fig. 1. Subject seated on the experimental position during a rotation movement.

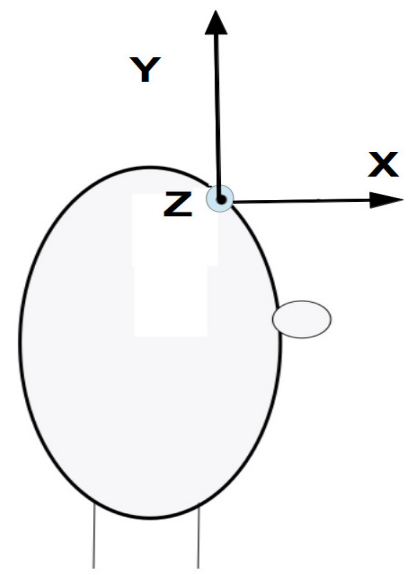

Fig. 2. Reference frame for the 3-D rotations.

in order to observe the clinical failure and not the efficiency of the medical treatment.

\subsection{Data analysis}

From the sensor physical data, fusion algorithms compute the 3-D rotation parameters at each sampling time. In particular, $i 4$ Motion ${ }^{\circledR}$ software provides an angle/axis representation calculated in the head reference frame at initial time $(\vec{X}, \vec{Y}, \vec{Z})$ (Fig. 2). We express the rotation axis in the frame corresponding to the ISB axis definition for the spine (Fig. 2) (Wu, 2002). The angle/axis model consists of four parameters at each sampling time: the resultant rotation axis orientation $\left(N_{X}, N_{Y}, N_{Z}\right)$ and the rotation angle around this axis $(\alpha)$.

The first step of the data analysis consists of computing the cardan angles according to defined cardan sequences. In a previous study, Garric, Portero, Masson, 
and Portero (2014) had automatically computed the rotation order for various subjects and for various primary movements with the Angle/Axis model. They had chosen the most frequent sequence based on the order of movement importance in the fixed head reference frame for each of the three primary movements in order to be able to compare all the subjects. Therefore, we have used:

i. for a primary movement of flexion-extension: the cardan sequence ZYX (associated movements were successfully axial rotation and lateral flexion),

ii. for a primary movement of axial rotation: the cardan sequence YXZ (associated movements were successfully lateral flexion and flexion-extension),

iii. for a primary movement of lateral flexion: the cardan sequence XYZ (associated movements were successfully axial rotation and flexion-extension).

Using these cardan sequences, data of the angle/axis representation, $(\vec{N}, \alpha)$ were firstly transformed in quaternion data, $Q=q_{w}+q_{x} i+q_{y} j+q_{z} k$, thanks to the formula:

$$
\begin{aligned}
q_{w} & =\cos (\alpha / 2) \\
q_{x} & =N_{x} \times \sin (\alpha / 2) \\
q_{y} & =N_{y} \times \sin (\alpha / 2) \\
q_{z} & =N_{z} \times \sin (\alpha / 2) .
\end{aligned}
$$

Then, the expected cardan angular values were obtained from the following general equations:

$$
\begin{aligned}
\tan \left(\theta_{1}\right) & =\frac{2\left(q_{w} q_{1}-e q_{2} q_{3}\right)}{1-2\left(q_{1}^{2}+q_{2}^{2}\right)} \\
\sin \left(\theta_{2}\right) & =2\left(q_{w} q_{2}+e q_{1} q_{3}\right) \\
\tan \left(\theta_{3}\right) & =\frac{2\left(q_{w} q_{3}-e q_{1} q_{2}\right)}{1-2\left(q_{2}^{2}+q_{3}^{2}\right)}
\end{aligned}
$$

where:

- $\theta_{1}, \theta_{2}$ and $\theta_{3}$ are successive rotation angles around the main axis $\vec{E}_{1}$, the second axis $\vec{E}_{2}$ and the third axis $\vec{E}_{3}$. The indices correspond to the cardan sequence order.

$-R=\left(\vec{E}_{1}, \vec{E}_{2}, \vec{E}_{3}\right)$ is a frame, associated with head position at time t0. For instance, considering the cardan sequence ZYX, we have: $\vec{E}_{1}=\vec{Z}=\vec{k}, \vec{E}_{2}=\vec{Y}=$ $\vec{j}, \vec{E}_{3}=\vec{X}=\vec{i}$.

- e is 1 if the frame $\left(\vec{E}_{3}, \vec{E}_{2}, \vec{E}_{1}\right)$ obey the righthand orientation rule (e.g. with sequences ZYX, XZY, YXZ) and e is -1 if the other cases.

- $q_{1}, q_{2}$ and $q_{3}$ depends on the cardan sequence expected. For instance, considering the cardan sequence ZYX, the quaternion must be: $Q=q_{w}+q_{3} i+q_{2} j+q_{1} k$.

All the computations described before were performed with Scilab 5.5.0 (Scilab Entreprises, Versailles, France).
Finally, numerical computations were made on the cardan curves. The head RoM was obtained by the difference between the maximal and minimal angular positions of the head sensor in each axis and during the three movement cycles. A graphical interface, developed with Matlab 7.5.0 (The Mathworks Inc., Natick, USA), allows an automatic treatment of the cardan sequences of each movement performed in order to calculate the RoMs (Fig. 3). The values of each associated RoM were normalized according to the RoM of the corresponding primary movement.

Reproducibility of head RoMs was defined by calculation of the standard error of measurement (SEM), and the intraclass correlation coefficient (ICC). An absolute correlation coefficient of 0.6 was considered as indicator of statistical significance (Sleivert \&Wenger, 1994). Bland and Altman graphs with accompanying 95\% limits of agreement, provided a visual representation of the RoM over the two testing sessions and depicted the difference of RoM (between test and retest) plotted according to the mean RoM of the two testing sessions for each participant. The 95\% upper and lower limits of agreement represented two s.d. more than and less than the mean difference of RoM.

Data collected with the sensor positioned on the trunk were only used to control if the ranges of the compensatory trunk movements were negligible enough for not affecting significantly the head kinematics data.

\section{Results}

In a first part concerning the test-retest reproducibility study, means \pm s.d. of RoMs for primary and associated movements and standard error of measurement (SEM) are indicated in (Tab. 1):

- For primary movements, the mean ICC was between 0.77 and 0.86 .

- ICCs were 0.61 and 0.77 for associated movements in the transverse plane.

- ICCs were 0.66 and 0.92 for associated movements in the frontal plane.

- For associated movements in the sagittal plane, ICCs were 0.28 and 0.49 .

Overall, the Bland and Altman plots confirmed the results obtained from the calculation of the ICCs (Fig. 4).

Means \pm s.d. values of the angular displacements for the sensor positioned on the trunk were $8.0 \pm 4.4^{\circ}$ and $5.4 \pm 3.8^{\circ}$ in flexion extension for $\mathrm{T} 1$ and $\mathrm{T} 2$, respectively. In axial rotation, they were $2.7 \pm 1.1^{\circ}$ and $2.9 \pm 1.8^{\circ}$ for $\mathrm{T} 1$ and T2, respectively. In lateral flexion, they were $3.7 \pm$ $2.3^{\circ}$ and $2.7 \pm 1.1^{\circ}$ for $\mathrm{T} 1$ and $\mathrm{T} 2$, respectively.

In a second part, concerning the clinical case study, cardan curves are given in Figure 5. For the primary movements and before the training, we found a maximal right axial rotation of $7^{\circ}$ and a maximal left axial rotation of $34^{\circ}$, for a RoM of $41^{\circ}$. After the training, the maximal 


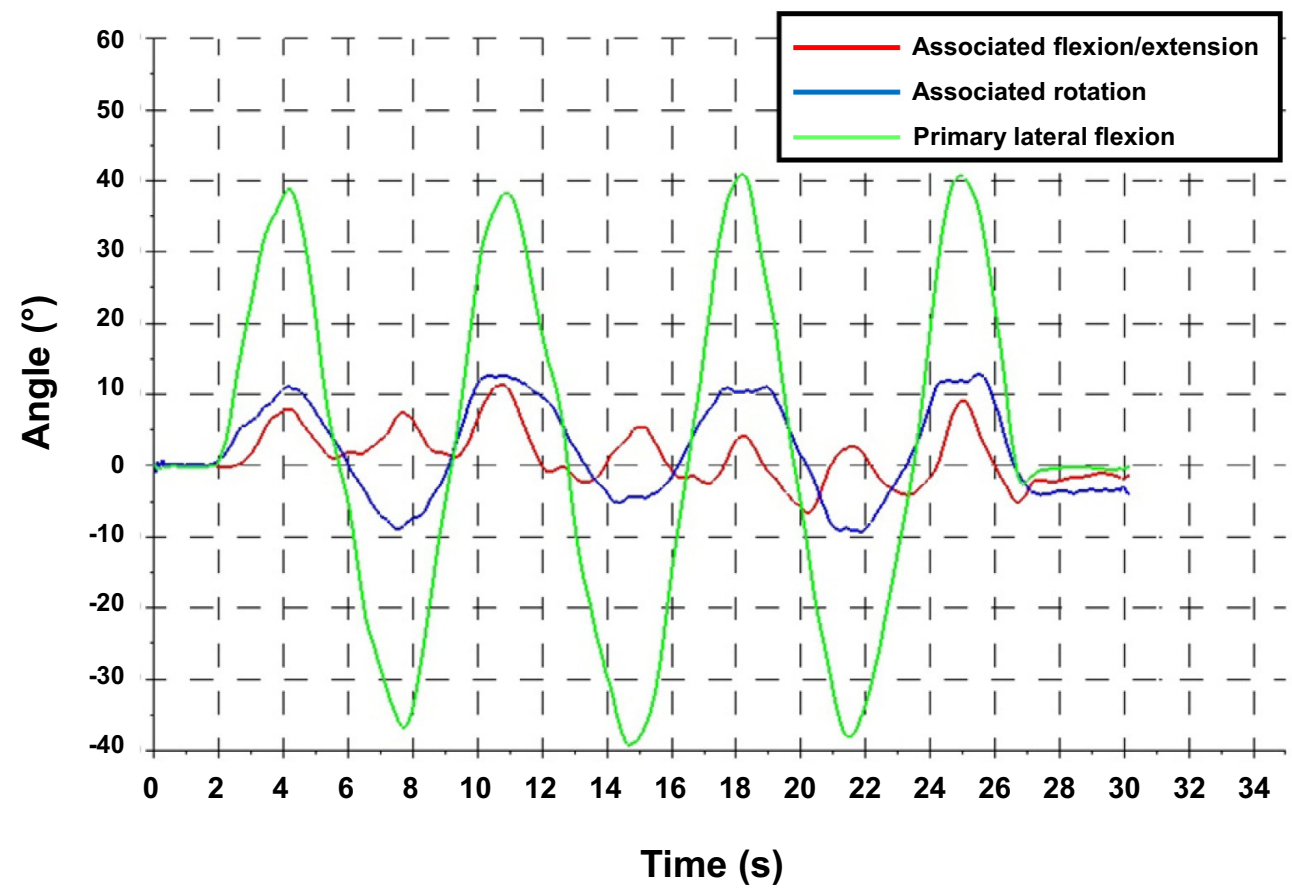

Fig. 3. Typical pattern of primary lateral flexion with associated ipsilateral rotation and associated flexion (convention: right axial rotation, right lateral flexion and flexion are positives). Head RoM was obtained by the difference between the maximal and minimal angular position of the head sensor in each axis during the three cycles of the movement.
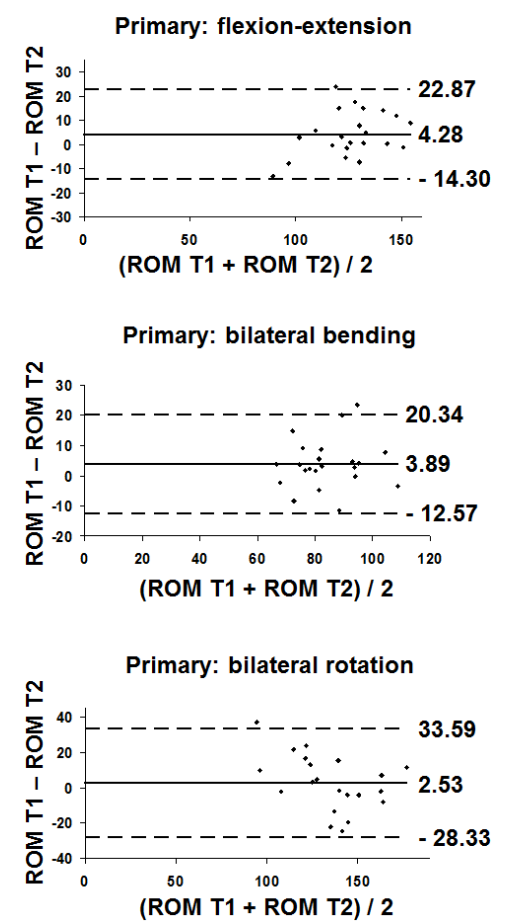
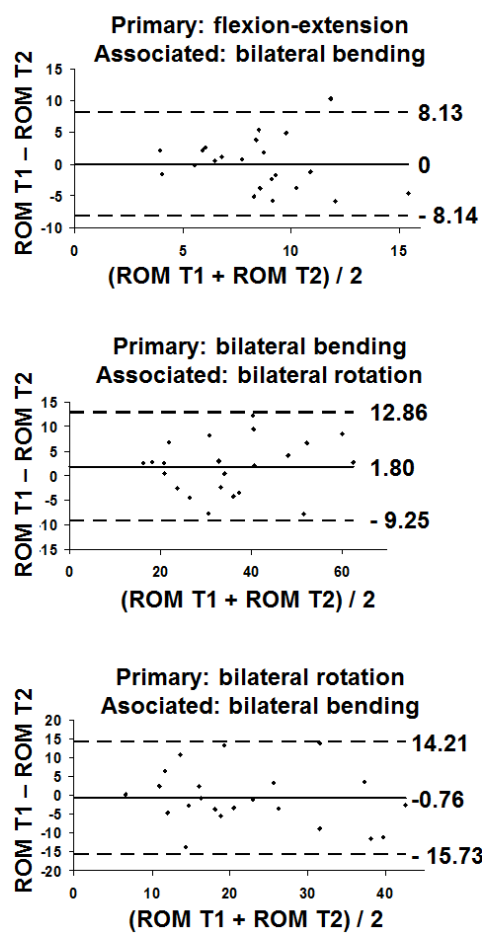
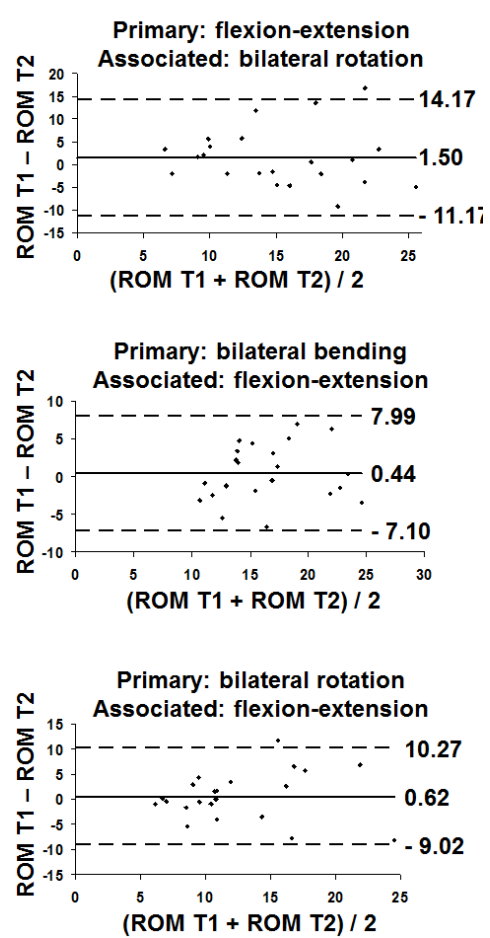

Fig. 4. Distribution plot from Bland and Altman tests for between-session reproducibility (T1 and T2) of primary and associated movements in the three anatomical planes. Bias (value of the mean of the differences) is represented by the full horizontal line. Limit of agreement (value of the mean of the differences $\pm 2 \mathrm{~s}$. d.) is represented by the horizontal dotted lines. 
Table 1. Means, s. d., m. d., relative values, and reproducibility of primary and associated RoMs in the three anatomical planes between $\mathrm{T} 1$ and $\mathrm{T} 2$.

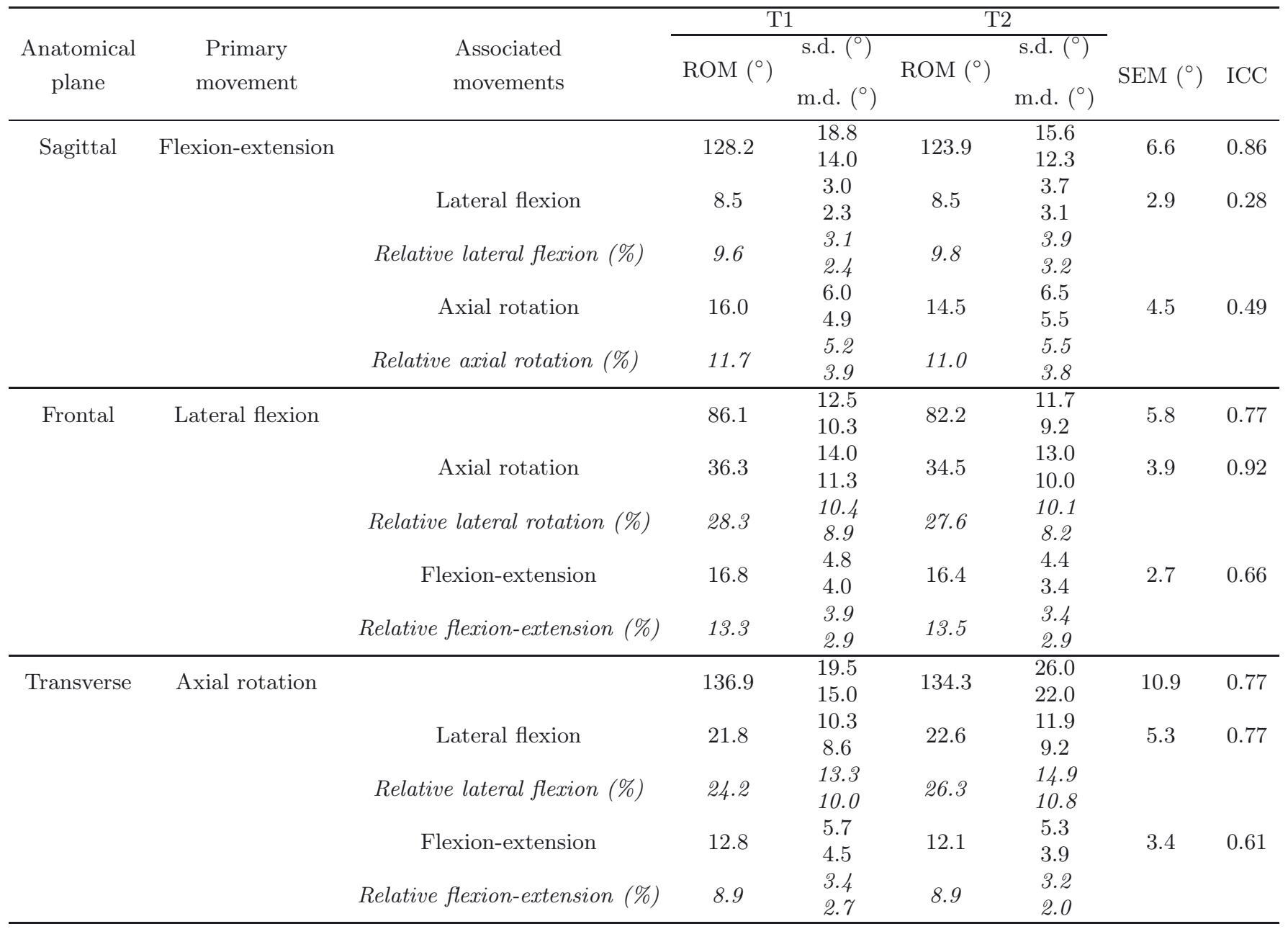

right axial rotation was $14^{\circ}$ and the maximal left axial rotation was $22^{\circ}$ for a RoM of $36^{\circ}$ (from Demaille-Wlodyka et al. (2007), physiological RoM for healthy subjects of the same gender and age is: $148.63 \pm 14.97^{\circ}$ ).

For the associated movements in the frontal plane and before the training, we found a maximal right lateral flexion of $15^{\circ}$ and a maximal left lateral flexion of $11^{\circ}$, for a RoM of $26^{\circ}$. After the training, the maximal right lateral flexion was $3^{\circ}$ and the maximal left lateral flexion was $15^{\circ}$ for a RoM of $18^{\circ}$. For the associated movements in the sagittal plane and before the training, we found a maximal flexion of $8^{\circ}$ and a maximal extension of $8^{\circ}$, for a RoM of $16^{\circ}$. After the training, the maximal flexion was $11^{\circ}$ and the maximal extension was $5^{\circ}$ for a RoM of $16^{\circ}$.

\section{Discussion}

The main goal of our study was to assess the reproducibility of head RoM of primary and associated movements estimated with a cardan method using inertial sensors through a reproducibility study. The maximal RoMs for the primary movements measured by this method were close to other results available in the literature (Tab. 2). In addition, concerning the values of associated movements, our results are similar to those available in the literature. For instance, Demaille-Wlodyka et al. (2007) noticed angular values for associated movements of $28.8^{\circ}$ in lateral flexion and $21.4^{\circ}$ in flexion-extension during primary movements in the transverse plane, $35.4^{\circ}$ in axial rotation and $18.6^{\circ}$ in flexion-extension during primary movements in the frontal plane, and $10.8^{\circ}$ in lateral flexion and $15.7^{\circ}$ in axial rotation for primary movements in the sagittal plane.

The statistical analysis allowed us to consider the head movement measures with the inertial sensors system as reproducible for primary movements and associated movements in the frontal and transverse planes. Even if our sample may induce some bias in the interpretation of statistical analysis (ICC increase with the heterogeneity of population (Xing, Madden, Duggan, \& Lyons, 2003)), the 
Table 2. Values of head RoMs in the three anatomical planes reported in the literature.

\begin{tabular}{|c|c|c|c|c|}
\hline Authors & Devices & $\begin{array}{l}\text { RoM rotation } \\
\text { (Means } \pm \text { s.d.) }\end{array}$ & $\begin{array}{c}\text { RoM lateral flexion } \\
\text { (Means } \pm \text { s.d. })\end{array}$ & $\begin{array}{c}\text { RoM flexion/extension } \\
\text { (Means } \pm \text { s.d. })\end{array}$ \\
\hline Feipel et al., 1999 & Electrogoniometry & $144 \pm 20^{\circ}$ & $88 \pm 16^{\circ}$ & $122 \pm 18^{\circ}$ \\
\hline $\begin{array}{c}\text { Jordan, Dzedzic, Jones, } \\
\text { Ong, \& Dawes, } 2000\end{array}$ & Electromagnetic tracking & $158.4 \pm 15.5^{\circ}$ & $90.9 \pm 14.4^{\circ}$ & $133.2 \pm 16.7^{\circ}$ \\
\hline Mannion, Klein, & Ultrasonography & $150.8 \pm 12.4^{\circ}$ & $85.1 \pm 13.4^{\circ}$ & $127.8 \pm 17.9^{\circ}$ \\
\hline Dvorak, \& Lanz, 2000 & Electrogoniometry & $144.3 \pm 12.3^{\circ}$ & $82.7 \pm 12.5^{\circ}$ & $118.8 \pm 16.7^{\circ}$ \\
\hline Malmström, Karlberg, Melander, & Ultrasonography & $155.3 \pm 14.4^{\circ}$ & $84 \pm 12.3^{\circ}$ & $137.7 \pm 15.7^{\circ}$ \\
\hline \& Magnusson, 2003 & Goniometry & $151.5 \pm 14.9^{\circ}$ & $82.5 \pm 12.4^{\circ}$ & $132.1 \pm 15.3^{\circ}$ \\
\hline \multirow{3}{*}{ Morphett et al., 2003} & Electromagnetic tracking & $152.51 \pm 17.71^{\circ}$ & $77.97 \pm 13.50^{\circ}$ & $107.08 \pm 16.78^{\circ}$ \\
\hline & CROM device & $140.29 \pm 15.08^{\circ}$ & $88.57 \pm 14.61^{\circ}$ & $117.25 \pm 22.83^{\circ}$ \\
\hline & Visual estimation & $155.89 \pm 22.98^{\circ}$ & $82.86 \pm 13.91^{\circ}$ & $116.25 \pm 18.59^{\circ}$ \\
\hline Demaille-Wlodyka et al., 2007 & Ultrasonography & $143.9 \pm 22.8^{\circ}$ & $87 \pm 21.6^{\circ}$ & $126.2 \pm 21.6^{\circ}$ \\
\hline Theobald et al., $2012^{*}$ & Inertial sensors & $120^{\circ}$ & $73^{\circ}$ & $93^{\circ}$ \\
\hline
\end{tabular}

* Means for the four tests.
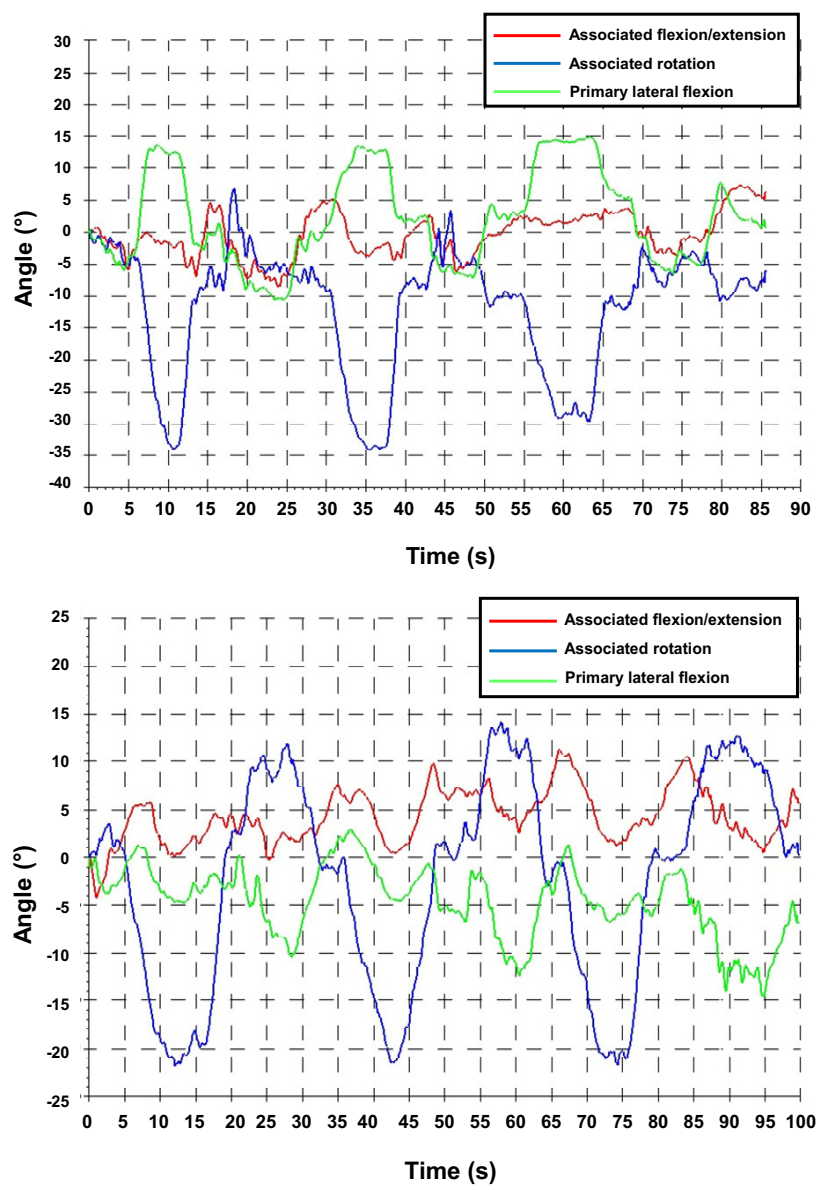

Fig. 5. A primary axial rotation movement (blue) with associated lateral flexion (green) and flexion/extension (red) before (up) and after (bottom) the self-training (convention: right axial rotation, right lateral flexion and flexion are positives).

results associated with values of RoMs highlight the reproducibility of our method except for associated movements in the sagittal plane. Indeed, RoM relative values of associated movements during primary flexion-extension seem weak in comparison with the other planes as suggested in previous studies (Demaille-Wlodyka et al., 2007; Feipel et al., 1999). We have also observed that the ranges of associated movements were low in these cases. We could hypothesize that associated movements in the sagittal plane are the consequence of postural adjustments during flexion-extension (which are unpredictable and difficult to reproduce) rather than the consequence of a primary movement component, generated mainly by the orientation of articular facets (at least for the lower part of the cervical spine) and muscle synergies.

This test-retest study presents limitations. Data computed from the thoracic sensor are used only to control that range of thoracic movements were negligible. In further studies, given additional kinematics data obtained in an external reference frame, rotation compositions will allow expressing the movements in the trunk reference frame.

Nevertheless, these preliminary test-retest reproducibility results of head RoMs confirm the significance of using the RoMP and the associated data treatment. Inertial sensors appear to be accurate tools to assess head movements. Thereafter, our method was applied on a clinical case study. Clinical changes could be observed for a given patient, confirming, so, the rehabilitation effects observed clinically. This clinical study needs to be further completed, it is nonetheless promising for clinical practice in order to evaluate patients with neck disorders (e.g. before/during/after rehabilitation), or elite athletes for career follow-up (e.g. sports with high risk neck injuries).

Acknowledgements. We thank the French Association for Research and Evaluation in Physiotherapy (AFREK) for its financial support for the clinical study.

\section{Bibliography}

Albanese, A., Bhatia, K., Bressman, S.B., DeLong, M.R., Fahn, S., Fung, V.S.C., Hallett, M., Jankovic, J., Jinnah, 
H.A., Klein, C., Lang, A.E., Mink, J.W., \& Teller, J.K. (2013). Phenomenology and classification of dystonia: a consensus update. Movement Disorders, 28, 863-873.

Audette, I., Dumas, J.-P., Côté, J.-N., \& De Serres, S.-J. (2010). Validity and between-day reliability of the cervical range of motion (CROM) device. The Journal of Orthopaedic and Sports Physical Therapy, 40, 318-323.

Begon, M., \& Lacouture, P. (2005). Modélisation anthropométrique pour une analyse mécanique du geste sportif, partie 2. Science et Motricité, 55 (2), 35-60.

Bertuit, J., Van Geyt, B., \& Feipel, V. (2008). Validité et fiabilité des moyens d'évaluation du rachis cervical : revue critique de la littérature. Kinésithérapie Scientifique, 1, $31-40$.

Bleton, J.-P. (2010) Physiotherapy of focal dystonia: a physiotherapist's personal experience. European Journal of Neurology, 17 (Suppl 1), 107-112.

Bonnechère, B., Salvia, P., Dugailly, P.-M., Maroye, L., Van Geyt, B., \& Feipel, V. (2014). Influence of movement speed on cervical range of motion. European Spine Journal, 23, 1688-1693.

Bouisson, L. (2008). Étude cinématique tridimensionnelle du rachis cervical. Comparaison entre sujets sains et pathologiques (thèse de doctorat, Université Claude Bernard - Lyon 1). http://tel.archives-ouvertes.fr/ tel-00367705.

Boussion, L., Bahuaud, P., \& Chèze, L. (2011). Proposal of a thorax segment coordinate system for the $3 \mathrm{D}$ kinematical analysis of the cervical spine. Computer Methods in Biomechanics and Biomedical Engineering, 14, 1041-1047.

Castro, W.H., Sautmann, A., Schilgen, M., \& Sautmann, M. (2000). Noninvasive three-dimensional analysis of cervical spine motion in normal subjects in relation to age and sex. An experimental examination. Spine, 25, 443-449.

Consky, E., Basinski, A., Belle, L., Ranawaya, R., \& Lang, A. (1990). The Toronto Western Spasmodic Torticollis Rating Scale (TWSTRS). Neurology, 40 (Suppl 1), 445.

Demaille-Wlodyka, S., Chiquet, C., Lavaste, J.-F., Skalli, W. Revel, M., \& Poiraudeau, S. (2007). Cervical range of motion and cephalic kinesthesis: ultrasonographic analysis by age and sex. Spine, 32, 254-261.

Duc, C., Salvia, P., Lubansu, A., Feipel, V., \& Aminian, K. (2013). Objective evaluation of cervical spine mobility after surgery during free-living activity. Clinical Biomechanics, 28, 364-369.

Dvir, Z., \& Prushansky, T. (2000). Reproducibility and instrument validity of a new ultrasonography-based system for measuring cervical spine kinematics. Clinical Biomechanics, 15, 658-664.

Feipel, V., Rondelet, B., Le Pallec, J.-P., \& Rooze, M. (1999). Normal global motion of the cervical spine: an electrogoniographic study. Clinical Biomechanics, 14, 462-470.

Garric, D., Portero, R., Masson, I., \& Portero, P. (2014). Vers la caractérisation des propriétés biomécaniques du segment tête-cou : utilisation de capteurs inertiels 3D-Première étape : étude cinématique. $1^{\text {ères }}$ Journées scientifiques franco-maghrébines : outils et méthodes de caractérisation mécanique des matériaux complexes, Hammamet, Tunisia.

Hallett, M. (2011). Neurophysiology of dystonia: The role of inhibition. Neurobiology of Disease, 42, 177-184.

Hopkins, W.G. (2000). Measures of reliability in sports medicine and science. Sports Medicine, 30, 1-15.

Jordan, K., Dziedzic, K., Jones, P.W., Ong, B.N., \& Dawes, P.T. (1992). The reliability of the three-dimensional FASTRAK measurement system in measuring cervical spine and shoulder range of motion in healthy subjects Rheumatology, 39, 382-388.

Karduna, A., McClure, P., \& Michener, L. (2000). Scapular kinematics: effects of altering the Euler angle sequence of rotations. Journal of Biomechanics, 33, 1063-1068.

Malmström, E.-M., Karlberg, M., Melander, A., \& Magnusson, M. (2003). Zebris versus Myrin: a comparative study between a three-dimensional ultrasound movement analysis and an inclinometer/compass method: intradevice reliability, concurrent validity, intertester comparison, intratester reliability, and intraindividual variability. Spine, 28, 433-440.

Mannion, A.F., Klein, G.N., Dvorak, J., \& Lanz, C. (2000). Range of global motion of the cervical spine: intraindividual reliability and the influence of measurement device. European Spine Journal 9, 379-385.

Marsh, W.A., Monroe, D.M., Brin, M.F., \& Gallagher, C.J. (2014). Systematic review and meta-analysis of the duration of clinical effect of onabotulinumtoxinA in cervical dystonia. BMC Neurology 14, 91.

McNair, P.J., Portero, P., Chiquet, C., Mawston, G., \& Lavaste, F. (2007). Acute neck pain: cervical spine range of motion and position sense prior to and after joint mobilization. Manual Therapy, 12, 390-394.

Morphett, A.L., Crawford, C.M., \& Lee, D. (2003). The use of electromagnetic tracking technology for measurement of passive cervical range of motion: a pilot study. Journal of Manipulative and Physiological Therapeutics, 26, 152-159.

Portero, P. (2009). Évaluation instrumentale de la mobilité et de la force musculaire du rachis cervical : applications cliniques. In S. Bendaya \& J.-C. Goussard (Eds.), Le rachis cervical vieillissant (pp. 21-29). Berlin: Springer.

Prushansky, T., \& Dvir, Z. (2008). Cervical motion testing: methodology and clinical implications. Journal of Manipulative Physiological Therapeutics, 31, 503-508.

Salvia, P., Champagne, O., Feipel, V., Rooze, M., \& de Beyl, D.Z. (2006). Clinical and goniometric evaluation of patients with spasmodic torticollis. Clinical Biomechanics, 21, 323-329. 
Sleivert, G.G., \& Wenger, H.A. (1994). Reliability of measuring isometric and isokinetic peak torque, rate of torque development, integrated electromyography, and tibial nerve conduction velocity. Archives of Physical Medicine and Rehabilitation, 75, 1315-1321.

Theobald, P.S., Jones, M.D., \& Williams, J.M. (2012). Do inertial sensors represent a viable method to reliably measure cervical spine range of motion? Manual Therapy, 17, 92-96.

Trott, P.H., Pearcy, M.J., Ruston, S.A., Fulton, I., \& Brien, C. (1996). Three-dimensional analysis of active cervical motion: the effect of age and gender. Clinical Biomechanics, 11, 201-206.

Watier, B. (2006). Comportement mécanique du rachis cervical : une revue de littérature. ITBM-RBM, 27, 92-106.
Wu, G. (2002). ISB recommendation on definitions of joint coordinate system of various joints for the reporting of human joint motion - part I: ankle, hip, and spine. Journal of Biomechanics, 35, 543-548.

Xing, Y., Madded, M.G., Duggan, J., \& Lyons, G.J. (2003). Distributed regression for heterogeneous data sets. In M.R. Berthold, H.J. Lenz, E. Bradley, R. Kruse, \& C. Borgelt (Eds.), Advances in intelligent data analysis $V$ (Lecture notes in computer science 2810) (pp. 544-553). Berlin: Springer.

Youdas, J.W., Garrett, T.R., Suman, V.J., Bogard, C.L., Hallman, H.O., \& Carey, J.R. (1992). Normal range of motion of the cervical spine: an initial goniometric study. Physical Therapy, 72, 770-780. 